SILVA, A. L.; RODRIGUES, J. A.; SILVA, G. O. A distribuição beta Fréchet transmutada: propriedades e aplicação

\title{
A DISTRIBUIÇÃO BETA FRÉCHET TRANSMUTADA: PROPRIEDADES E APLICAÇÃO A DADOS DE SOBREVIVÊNCIA
}

\author{
Ângela Lima da Silva ${ }^{1}$ \\ Jailson Rodrigues ${ }^{2}$ \\ Giovana Silva ${ }^{3}$
}

\begin{abstract}
RESUMO
Neste artigo, é definida e estudada uma distribuição com cinco parâmetros denominada beta Fréchet transmutada, além de serem apresentadas algumas das principais propriedades desse modelo. Em particular, são encontradas expressões para os momentos e para a função geradora de momentos. $\mathrm{O}$ método da máxima verossimilhança é empregado para estimar os parâmetros da distribuição. Um conjunto de dados é utilizado no ajuste da distribuição beta Fréchet transmutada e os resultados obtidos indicaram que o modelo pode oferecer um ajuste melhor que o de distribuições já consolidadas na análise de dados de sobrevivência.
\end{abstract}

Palavras-chave: Comparação de Modelos. Distribuição Fréchet. Método da Máxima Verossimilhança.

\section{THE BETA TRANSMUTED FRÉCHET DISTRIBUTION: PROPERTIES AND APPLICATION}

\begin{abstract}
In this article, is defined and studied a distribution with five parameters called beta Fréchet transmuted. Some of the main properties of this model are presented. In particular, expressions for the moments and moment generating function are deduced. The method of maximum likelihood is used to estimate the parameters of the distribution. A survival data set was used in the fit of distribution and the results indicated that the beta transmuted Fréchet model can offer a better fit than already consolidated distributions.

\footnotetext{
${ }^{1}$ Licenciada em Matemática pelo Instituto Federal da Bahia - IFBA, mestranda em Estatística na Universidade Federal da Bahia - UFBA. E-mail: angelalimadasilva@yahoo.com.br. Currículo Lattes: http://lattes.cnpq.br/1582007949170364.

${ }^{2}$ Licenciado em Matemática pela Universidade Estadual de Feira de Santana, mestre em Matemática pela UFBA e doutor em Estatística e Experimentação Agropecuária pela Universidade Federal de Lavras - UFLA. Professor adjunto do IFBA. E-mail: jailsondearaujo@yahoo.com.br.

Currículo Lattes: http://buscatextual.cnpq.br/buscatextual/visualizacv.do?id=K4184012J7.

${ }^{3}$ Graduada em Estatística pela Universidade Federal do Espírito Santo - UFES, mestrado em Estatística pela Universidade Federal do Rio de Janeiro - UFRJ e doutora em Estatística e Experimentação Agrícola pela ESALQ - USP. Professora adjunta do Departamento de Estatística da UFBA. E-mail: giovana.os@hotmail.com. Currículo Lattes: http://lattes.cnpq.br/3588724844050512.
} 
SILVA, A. L.; RODRIGUES, J. A.; SILVA, G. O. A distribuição beta Fréchet transmutada: propriedades e aplicação

Keywords: Comparison of Models. Fréchet Distribution. Maximum Likelihood Method.

\section{INTRODUÇÃO}

As distribuições de probabilidade são amplamente utilizadas para descrever fenômenos das diversas áreas do conhecimento. Esse fato é um catalisador que torna o estudo das distribuições de probabilidade um dos grandes temas de pesquisa em Estatística.

Nas últimas décadas, diversas classes de distribuições generalizadas foram propostas e estudadas. O interesse dessas propostas é desenvolver modelos mais flexíveis e com propriedades robustas que possam ser aplicados na descrição de variados fenômenos.

O objetivo deste trabalho é introduzir e estudar uma nova distribuição com cinco parâmetros, denominada beta Fréchet transmutada. Dessa forma, primeiramente serão apresentadas a construção do modelo e algumas propriedades. A seguir, será utilizado o método da máxima verossimilhança para ajustá-lo a um conjunto de dados de sobrevivência. A qualidade de ajuste é comparada a de outras distribuições para evidenciar a adequação da nova distribuição.

\section{REVISÃO TEÓRICA}

A distribuição Fréchet ou Weibull inversa, como também é conhecida, foi originalmente proposta por Keller, Kamath e Perera (1982) para analisar dados de confiabilidade e para investigar falhas de componentes mecânicos sujeitos à degradação. Com o decorrer dos anos, a distribuição Fréchet passou a ser um dos modelos mais utilizados na análise de dados de sobrevivência, o que levou ao surgimento de generalizações e extensões dessa distribuição. Baharith et al. (2014), introduziram uma generalização da distribuição Fréchet denominada distribuição beta Weibull inversa generalizada. Foram deduzidas importantes propriedades do modelo como, por exemplo, os momentos e a função geradora de momento. Além disso, o ajuste do modelo a um conjunto de dados de sobrevivência indicou a versatilidade do modelo.

Pararai, Warahena-Liyanage e Oluyede (2014) construíram uma generalização da distribuição Fréchet a partir da família gama-G, proposta por Ristic e Balakrishnan (2011). Essa nova distribuição foi chamada de gama Weibull inversa generalizada. Algumas propriedades desse modelo foram deduzidas, incluindo os momentos e os desvios médios. 
SILVA, A. L.; RODRIGUES, J. A.; SILVA, G. O. A distribuição beta Fréchet transmutada: propriedades e aplicação

Além disso, foi utilizado o método da máxima verossimilhança para ajustar os parâmetros dessa distribuição a um conjunto de dados de sobrevivência.

Khan e King (2012) propuseram a distribuição Weibull inversa modificada e discutiram suas várias propriedades. Em particular, foram obtidas expressões para os momentos, estatísticas de ordem e a matriz de informação de Fischer. Shahbaz, Shahbaz e Butt (2012) definiram e estudaram uma generalização da distribuição Fréchet com quatro parâmetros. Foram deduzidos os momentos, a função quantil e as equações de máxima verossimilhança. Gusmão, Ortega e Cordeiro (2011) introduziram a distribuição Fréchet generalizada com três parâmetros com função de risco decrescente e unimodal. Khan (2010) propôs a distribuição beta Weibull inversa. Algumas das principais propriedades dessa distribuição foram deduzidas pelo autor.

\section{METODOLOGIA}

\subsection{DADOS}

A tuberculose é uma doença que representa um sério problema de saúde pública mundial. De acordo com Sant'Anna e Hijjar (2007), no Brasil, estima-se a ocorrência de 111.000 novos casos de tuberculose por ano e cerca de 6.000 óbitos anuais.

No estudo dessa patologia são utilizadas cobaias. As distribuições probabilísticas são empregadas para descrever o tempo de sobrevivência de cobaias infectadas. Neste trabalho, foi analisado um conjunto de dados que corresponde aos tempos de sobrevivência, em dias, de 72 cobaias infectadas com diferentes doses do bacilo da tuberculose. Esses dados foram retirados de Bjerkedal (1960) e podem ser visualizados na Tabela 1.

Tabela 1- Tempos de sobrevivência das cobaias infectadas

\begin{tabular}{rrrrrrrrr}
\hline 12 & 15 & 22 & 24 & 24 & 32 & 32 & 33 & 34 \\
\hline 38 & 38 & 43 & 44 & 48 & 52 & 53 & 54 & 54 \\
54 & 56 & 57 & 58 & 58 & 59 & 60 & 60 & 60 \\
60 & 61 & 62 & 63 & 65 & 65 & 67 & 68 & 70 \\
70 & 72 & 73 & 75 & 76 & 76 & 81 & 83 & 84 \\
85 & 87 & 91 & 95 & 96 & 98 & 99 & 109 & 110 \\
121 & 127 & 129 & 131 & 143 & 146 & 146 & 175 & 175 \\
211 & 233 & 258 & 258 & 263 & 297 & 341 & 341 & 376 \\
\hline
\end{tabular}

Fonte: Bjerkedal (1960). 
SILVA, A. L.; RODRIGUES, J. A.; SILVA, G. O. A distribuição beta Fréchet transmutada: propriedades e aplicação

\subsection{MODELO PROPOSTO}

A função de distribuição acumulada (FDA) e a função densidade de probabilidade (FDP) da distribuição Fréchet são dadas, respectivamente, por:

$$
W(x)=\exp \left[-\left(\frac{\sigma}{x}\right)^{v}\right]
$$

e

$$
w(x)=v \sigma^{v} x^{-(v+1)} \exp \left[-\left(\frac{\sigma}{x}\right)^{v}\right]
$$

sendo que $x>0, \sigma>0, v>0$.

Se $G(x)$ é uma FDA de uma variável aleatória $X$. Então, a FDA de uma classe de funções de distribuições generalizadas é dada por:

$$
F(x)=\frac{1}{B(\alpha, \beta)} \int_{0}^{G(x)} t^{\alpha-1}(1-t)^{\beta-1} d t
$$

sendo que $B(\alpha, \beta)$ representa a função beta dada por:

$$
B(\alpha, \beta)=\int_{0}^{1} t^{\alpha-1}(1-t)^{\beta-1} d t
$$

A correspondente FDP a (3) é dada por:

$$
f(x)=\frac{1}{B(\alpha, \beta)} g(x) G(x)^{\alpha-1}(1-G(x))^{\beta-1},
$$

sendo que $g(x)=d G(x) / d x$

Segundo Mahmoud e Mandouh (2013), uma variável aleatória $X>0$ possui distribuição Fréchet transmutada com parâmetros $\sigma>0, v>0$ e $|\lambda| \leq 1$ quando sua FDA é dada por:

$$
G(x)=\left\{1+\lambda-\lambda \exp \left[-\left(\frac{\sigma}{x}\right)^{v}\right]\right\} \exp \left[-\left(\frac{\sigma}{x}\right)^{v}\right]
$$


SILVA, A. L.; RODRIGUES, J. A.; SILVA, G. O. A distribuição beta Fréchet transmutada: propriedades e aplicação

Observe que a distribuição Fréchet é um caso especial de (6) quando $\lambda=0$. Substituindo (6) em (3), obtém-se uma nova distribuição chamada de beta Fréchet transmutada (BFT) com FDA dada por:

$$
F(x)=\frac{B\left(\left\{1+\lambda-\lambda \exp \left[-\left(\frac{\sigma}{x}\right)^{v}\right]\right\} \exp \left[-\left(\frac{\sigma}{x}\right)^{v}\right] ; \alpha, \beta\right)}{B(\alpha, \beta)}
$$

A FDP associada ao modelo BFT é dada por:

$$
\begin{aligned}
& f(x)=\frac{v}{\sigma B(\alpha, \beta)}\left(\frac{\sigma}{x}\right)^{v+1}\left\{1+\lambda-2 \lambda \exp \left[-\left(\frac{\sigma}{x}\right)^{v}\right]\right\} \times\left\{1+\lambda-\lambda \exp \left[-\left(\frac{\sigma}{x}\right)^{v}\right]\right\}^{\alpha-1} \exp \left[-\alpha\left(\frac{\sigma}{x}\right)^{v}\right] \\
& \times\left(1-\left\{1+\lambda-\lambda \exp \left[-\left(\frac{\sigma}{x}\right)^{v}\right]\right\} \exp \left[-\left(\frac{\sigma}{x}\right)^{v}\right]\right)^{\beta-1}
\end{aligned}
$$

No Gráfico 1 são exibidas algumas formas possíveis da FDP (8) para diferentes valores dos parâmetros $\alpha, \beta, \sigma, v$ e $\lambda$.

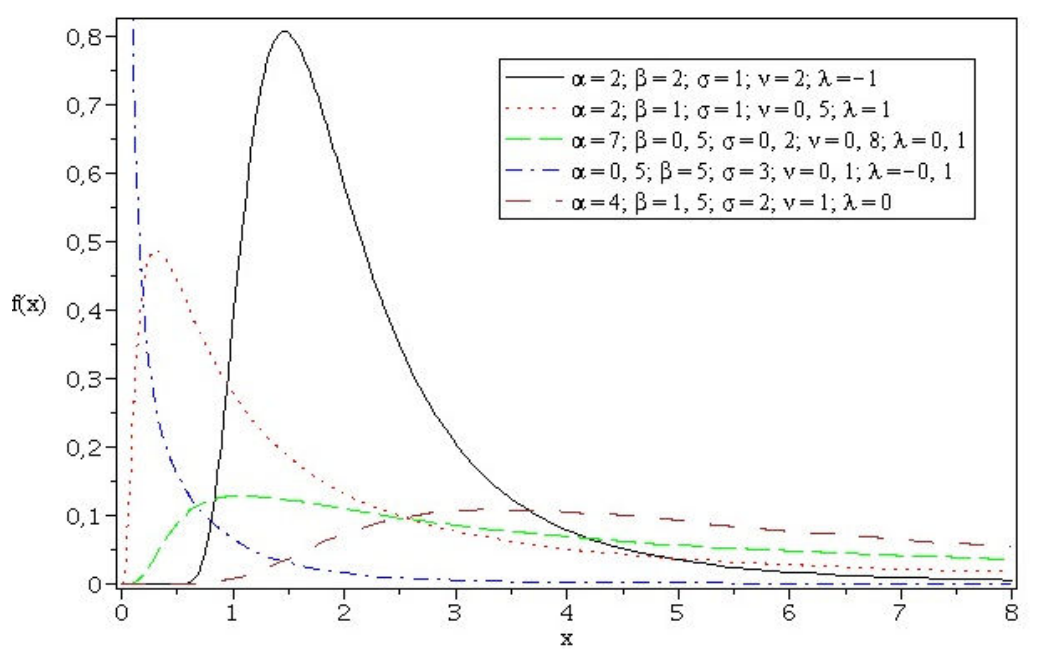

Gráfico 1- Gráficos da FDP da distribuição BFT para diferentes valores paramétricos Fonte: Autor.

A distribuição BFT apresenta alguns submodelos importantes.

1) Se $\beta=1$, o modelo BFT reduz-se a distribuição Fréchet transmutada exponenciada; 
SILVA, A. L.; RODRIGUES, J. A.; SILVA, G. O. A distribuição beta Fréchet transmutada: propriedades e aplicação

2) Quando $\alpha=\beta=1$, tem-se a distribuição Fréchet transmutada;

3) Caso $\beta=1$ e $\lambda=0$, o modelo BFT representa a distribuição Fréchet generalizada;

4) Quando $\alpha=\beta=1$ e $\lambda=0$, tem-se o modelo Fréchet;

5) Se $\alpha=\beta=1, \lambda=0$ e $v=1$, então a distribuição BFT reduz-se a distribuição exponencial inversa $(\mathrm{EI})$;

6) Caso $\alpha=\beta=1, \lambda=0$ e $v=2$, então a distribuição BFT representa o modelo Rayleigh inverso (RI) introduzido por Vodã (1972).

Para todo número real não inteiro $\beta>0$, pode-se escrever a seguinte expansão em potências:

$$
(1-z)^{\beta-1}=\sum_{j=0}^{\infty} \frac{(-1)^{j} \Gamma(\beta)}{\Gamma(\beta-j) j !} z^{j}
$$

sendo que $|z|<1$ e $\Gamma(\alpha)$ representa a função gama dada por:

$$
\Gamma(\alpha)=\int_{0}^{\infty} t^{\alpha-1} \exp (-t) d t
$$

Utilizando a representação (9) em (7), obtém-se a seguinte representação para FDA da distribuição BFT:

$$
F(x)=\frac{\Gamma(\alpha+\beta)}{\Gamma(\alpha)} \sum_{j=0}^{\infty} \sum_{k=0}^{\infty} \frac{(-1)^{j+k} \Gamma(\alpha+j)(1+\lambda)^{\alpha+j}}{\Gamma(\beta-j) \Gamma(\alpha+j+1-k) j ! k !} \times\left(\frac{\lambda}{1+\lambda}\right)^{k} \exp \left[-(\alpha+j+k)\left(\frac{\sigma}{x}\right)^{v}\right]
$$

Se $\beta>0$ é um inteiro, então, o índice $j$ para em $\beta-1$. Se $\alpha>0$ é um inteiro, então, índice $k$ para em $\alpha+j$. Diferenciando (11) com relação à $x$, pode-se reescrever a FDP da distribuição BFT da seguinte forma:

$$
f(x)=\frac{\Gamma(\alpha+\beta)}{\Gamma(\alpha)} \sum_{j=0}^{\infty} \sum_{k=0}^{\infty} \frac{(-1)^{j+k} \Gamma(\alpha+j)(1+\lambda)^{\alpha+j} v}{\Gamma(\beta-j) \Gamma(\alpha+j+1-k) j ! k !} \times \frac{\alpha+j+k}{\sigma}\left(\frac{\sigma}{x}\right)^{v+1}\left(\frac{\lambda}{1+\lambda}\right)^{k} \exp \left[-(\alpha+j+k)\left(\frac{\sigma}{x}\right)^{v}\right]
$$


SILVA, A. L.; RODRIGUES, J. A.; SILVA, G. O. A distribuição beta Fréchet transmutada: propriedades e aplicação

O momento de ordem $r$ da distribuição BFT é dado por:

$$
E\left(x^{r}\right)=\frac{\Gamma(\alpha+\beta)}{\Gamma(\alpha)} \sum_{j=0}^{\infty} \sum_{k=0}^{\infty} \frac{(-1)^{j+k} \Gamma(\alpha+j)(1+\lambda)^{\alpha+j}}{\Gamma(\beta-j) \Gamma(\alpha+j+1-k) j ! k !} \times \Gamma\left(1-\frac{r}{v}\right) \sigma^{r}(\alpha+j+k)_{v}^{\frac{r}{v}}\left(\frac{\lambda}{1+\lambda}\right)^{k}
$$

sempre que $v>r$.

A função geradora de momentos do modelo BFT é dada por:

$$
M(t)=E(\exp (t X))=\frac{\Gamma(\alpha+\beta)}{\Gamma(\alpha)} \sum_{j=0}^{\infty} \sum_{k=0}^{\infty} \sum_{r=0}^{\infty} \frac{(-1)^{j+k} t^{r} \Gamma(\alpha+j)(1+\lambda)^{\alpha+j}}{\Gamma(\beta-j) \Gamma(\alpha+j+1-k) j ! k ! r !} \Gamma\left(1-\frac{r}{v}\right) \sigma^{r}(\alpha+j+k)_{v}^{\frac{r}{v}}\left(\frac{\lambda}{1+\lambda}\right)^{k}
$$

sempre que $|t|<1$.

\subsection{INFERÊNCIA DOS PARÂMETROS}

A estimação dos parâmetros da distribuição BFT será feita utilizando o método da máxima verossimilhança. Suponha que $X_{1}, X_{2}, \ldots, X_{n}$ é uma amostra aleatória de tamanho $n$ da distribuição BFT. A função de log-verossimilhança correspondente é dada por:

$$
\begin{gathered}
\log L(\alpha, \beta, \sigma, v, \lambda)=n \log v-n \log \sigma+n \log \Gamma(\alpha+\beta) \\
+n(v+1) \log \sigma-n \log \Gamma(\alpha)-n \log \Gamma(\beta)-\alpha \sum_{j=1}^{n}\left(\frac{\sigma}{x_{j}}\right)^{v} \\
-(v+1) \sum_{j=1}^{n} \log x_{j}+\sum_{j=1}^{n} \log \left(1+\lambda-2 \lambda \exp \left[-\left(\frac{\sigma}{x_{j}}\right)^{v}\right]\right)+(\beta-1) \sum_{j=1}^{n} \log \left(1-\left\{1+\lambda-\lambda \exp \left[-\left(\frac{\sigma}{x_{j}}\right)^{v}\right]\right\} \exp \left[-\left(\frac{\sigma}{x_{j}}\right)^{v}\right]\right) \\
+(\alpha-1) \sum_{j=1}^{n} \log \left(1+\lambda-\lambda \exp \left[-\left(\frac{\sigma}{x_{j}}\right)^{v}\right]\right)
\end{gathered}
$$

Diferenciando (15) em relação a cada um dos parâmetros $\alpha, \beta, \sigma, v, \lambda$ e igualando os resultados a zero, obtém-se as seguintes equações de máximo verossimilhança: 
SILVA, A. L.; RODRIGUES, J. A.; SILVA, G. O. A distribuição beta Fréchet transmutada: propriedades e aplicação

$$
\begin{aligned}
& \frac{\partial \log L}{\partial \alpha}=n[\psi(\alpha+\beta)-\psi(\alpha)]-\sum_{j=1}^{n}\left(\frac{\sigma}{x_{j}}\right)^{v}+\sum_{j=1}^{n} \log \left(1+\lambda-\lambda \exp \left[-\left(\frac{\sigma}{x_{j}}\right)^{v}\right]\right) \\
& \frac{\partial \log L}{\partial \beta}=n[\psi(\alpha+\beta)-\psi(\beta)]+\sum_{j=1}^{n} \log \left(1-\left\{1+\lambda-\lambda \exp \left[-\left(\frac{\sigma}{x_{j}}\right)^{v}\right]\right\} \exp \left[-\left(\frac{\sigma}{x_{j}}\right)^{v}\right]\right) \\
& \frac{\partial \log L}{\partial \sigma}=\frac{n v}{\sigma}-\alpha v \sum_{j=1}^{n} x_{j}\left(\frac{\sigma}{x_{j}}\right)^{v+1}+\sum_{j=1}^{n} \frac{2 v \lambda x_{j}\left(\frac{\sigma}{x_{j}}\right)^{v+1} \exp \left[-\left(\frac{\sigma}{x_{j}}\right)^{v}\right]}{1+\lambda-2 \lambda \exp \left[-\left(\frac{\sigma}{x_{j}}\right)^{v}\right]} \\
& +(\beta-1) \sum_{j=1}^{n} \frac{v x_{j}\left(1+\lambda-2 \lambda \exp \left[-\left(\frac{\sigma}{x_{j}}\right)^{v}\right]\right)\left(\frac{\sigma}{x_{j}}\right)^{v+1} \exp \left[-\left(\frac{\sigma}{x_{j}}\right)^{v}\right]}{1-\left\{1+\lambda-\lambda \exp \left[-\left(\frac{\sigma}{x_{j}}\right)^{v}\right]\right\} \exp \left[-\left(\frac{\sigma}{x_{j}}\right)^{v}\right]} \\
& +(\alpha-1) \sum_{j=1}^{n} \frac{v \lambda x_{j}\left(\frac{\sigma}{x_{j}}\right)^{v+1} \exp \left[-\left(\frac{\sigma}{x_{j}}\right)^{v}\right]}{1+\lambda-\lambda \exp \left[-\left(\frac{\sigma}{x_{j}}\right)^{v}\right]} \\
& \frac{\partial \log L}{\partial v}=\frac{n}{v}+n \log v-\alpha \sum_{j=1}^{n}\left(\frac{\sigma}{x_{j}}\right)^{v} \log \left(\frac{\sigma}{x_{j}}\right)-\sum_{j=1}^{n} \log x_{j}+(\beta-1) \sum_{j=1}^{n} \frac{\left(1+\lambda-2 \lambda \exp \left[-\left(\frac{\sigma}{x_{j}}\right)\right]\right)\left(\frac{\sigma}{x_{j}}\right)^{v} \exp \left[-\left(\frac{\sigma}{x_{j}}\right)^{v}\right] \log \left(\frac{\sigma}{x_{j}}\right)}{1-\left\{1+\lambda-\lambda \exp \left[-\left(\frac{\sigma}{x_{j}}\right)^{v}\right]\right\} \exp \left[-\left(\frac{\sigma}{x_{j}}\right)^{v}\right]} \\
& +(\beta-1) \sum_{j=1}^{n} \frac{\left(1+\lambda-2 \lambda \exp \left[-\left(\frac{\sigma}{x_{j}}\right)\right]\left(\frac{\sigma}{x_{j}}\right)^{v} \exp \left[-\left(\frac{\sigma}{x_{j}}\right)^{v}\right] \log \left(\frac{\sigma}{x_{j}}\right)\right.}{1-\left\{1+\lambda-\lambda \exp \left[-\left(\frac{\sigma}{x_{j}}\right)^{v}\right]\right\} \exp \left[-\left(\frac{\sigma}{x_{j}}\right)^{v}\right]}+\lambda(\alpha-1) \sum_{j=1}^{n} \frac{\left(\frac{\sigma}{x_{j}}\right)^{v} \exp \left[-\left(\frac{\sigma}{x_{j}}\right)^{v}\right] \log \left(\frac{\sigma}{x_{j}}\right)}{1+\lambda-\lambda \exp \left[-\left(\frac{\sigma}{x_{j}}\right)^{v}\right]} \\
& +2 \lambda \sum_{j=1}^{n} \frac{\left(\frac{\sigma}{x_{j}}\right)^{v} \exp \left[-\left(\frac{\sigma}{x_{j}}\right)^{v}\right] \log \left(\frac{\sigma}{x_{j}}\right)}{1+\lambda-2 \lambda \exp \left[-\left(\frac{\sigma}{x_{j}}\right)^{v}\right]} \\
& \frac{\partial \log L}{\partial \lambda}=(\alpha-1) \sum_{j=1}^{n} \frac{1-\exp \left[-\left(\frac{\sigma}{x_{j}}\right)\right]}{1+\lambda-\lambda \exp \left[-\left(\frac{\sigma}{x_{j}}\right)\right]}+(1-\beta) \sum_{j=1}^{n} \frac{\left\{1-\exp \left[-\left(\frac{\sigma}{x_{j}}\right)^{v}\right]\right\} \exp \left[-\left(\frac{\sigma}{x_{j}}\right)^{v}\right]}{1-\left\{1+\lambda-\lambda \exp \left[-\left(\frac{\sigma}{x_{j}}\right)^{v}\right]\right\} \exp \left[-\left(\frac{\sigma}{x_{j}}\right)^{v}\right]}
\end{aligned}
$$

ForSci.: r. cient. IFMG campus Formiga, Formiga, v. 3, n. 1, p. 57-69, jan./jun. 2015. 
SILVA, A. L.; RODRIGUES, J. A.; SILVA, G. O. A distribuição beta Fréchet transmutada: propriedades e aplicação

$+\sum_{j=1}^{n} \frac{1-2 \exp \left[-\left(\frac{\sigma}{x_{j}}\right)^{v}\right]}{1+\lambda-2 \lambda \exp \left[-\left(\frac{\sigma}{x_{j}}\right)^{v}\right]}$

As estimativas de máxima verossimilhança $\hat{\alpha}, \hat{\beta}, \hat{\sigma}, \hat{v}, \hat{\lambda}$ dos cinco parâmetros $\alpha, \beta, \sigma, v \mathrm{e} \lambda$ são obtidas resolvendo simultaneamente as Equações não lineares (16)-(20). Para isso, utiliza-se um algoritmo quasi-Newton implementado no programa $\mathrm{R}$ ( $\mathrm{R}$ DEVELOPMENT CORE TEAM, 2011).

\section{RESULTADOS}

O ajuste do modelo BFT foi comparado com outras quatro distribuições: EI, Fréchet, RI e Weibull. Na Tabela 2, podem ser observadas as estimativas de máxima verossimilhança encontradas nos ajustes dessas distribuições.

Tabela 2 - Estimativas dos parâmetros das distribuições BFT, EI, Fréchet, RI, Weibull e AIC

\begin{tabular}{lcccccc}
\hline Modelo & $\hat{\alpha}$ & $\hat{\beta}$ & $\hat{\sigma}$ & $\hat{v}$ & $\hat{\lambda}$ & AIC \\
\hline BFT & 33,201 & 2,292 & 3,014 & 0,909 & $-0,042$ & 794,8 \\
EI & 1 & 1 & 60,098 & 1 & 0 & 807,3 \\
Fréchet & 1 & 1 & 54,183 & 1,415 & 0 & 795.3 \\
RI & 1 & 1 & 46,775 & 2 & 0 & 815.5 \\
Weibull & $\ldots$. & $\ldots .$. & 0,009 & 1,392 & 0 & 798,3 \\
\hline
\end{tabular}

Fonte: Autor.

Os valores do Critério de Informação de Akaike (AIC) que foram obtidos para as distribuições ajustadas são apresentados na Tabela 2. Os resultados indicam que a distribuição BFT apresentou um ajuste significativamente melhor que os outros quatro modelos.

No Gráfico 2, pode-se notar que, com exceção da distribuição RI, as demais curvas de ajustes acompanharam as condições de moda e assimetria, não sendo possível determinar, visualmente, a que melhor se ajustou aos dados observados. 
SILVA, A. L.; RODRIGUES, J. A.; SILVA, G. O. A distribuição beta Fréchet transmutada: propriedades e aplicação

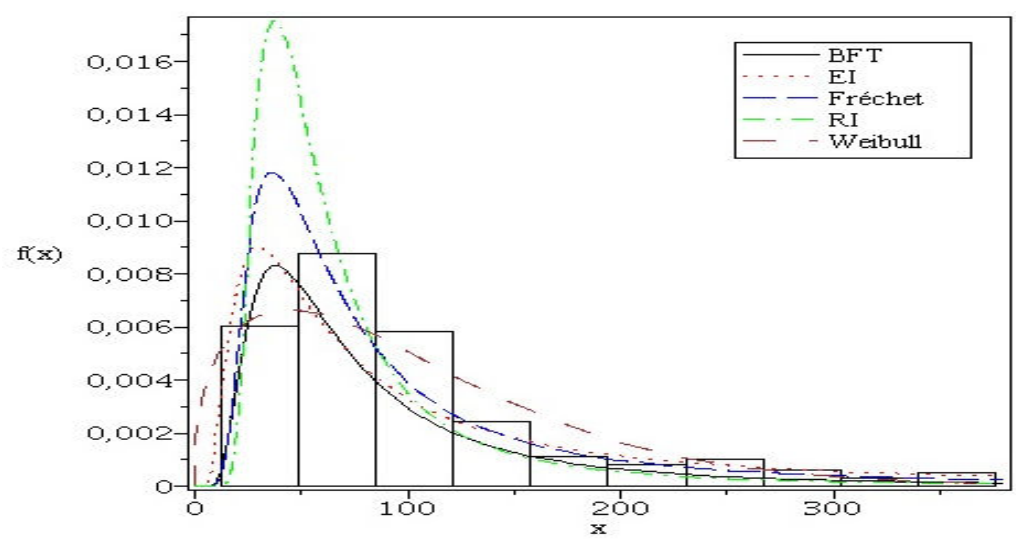

Gráfico 2 - Histograma de frequência dos dados observados sobreposto pelas FDP ajustadas BFT, EI, Fréchet, RI e Weibull Fonte: Autor.

Nos Gráficos de 3 a 7 são apresentados os gráficos de probabilidade para os cinco modelos avaliados. Ao observar o gráfico, pode-se notar que a distribuição BFT apresentou melhor ajuste em relação às demais distribuições e o pior o ajuste foi apresentado pelo modelo RI.

Dessa forma, os gráficos de probabilidade corroboram com os resultados obtidos anteriormente na análise dos valores de AIC.

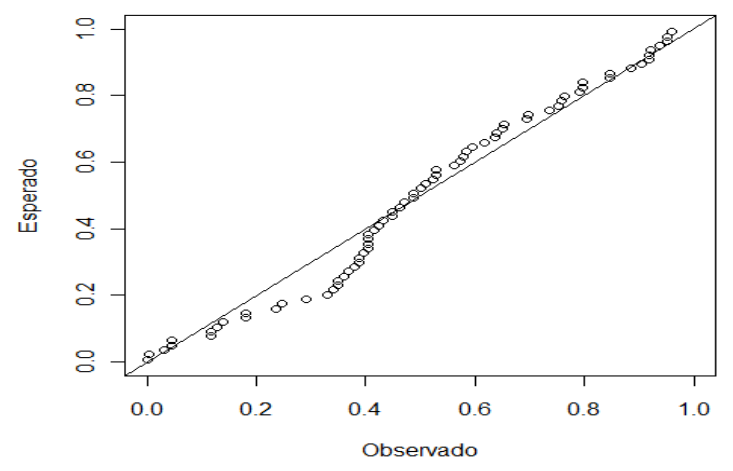

Gráfico 3 - Gráfico de probabilidade BFT Fonte: Autor.

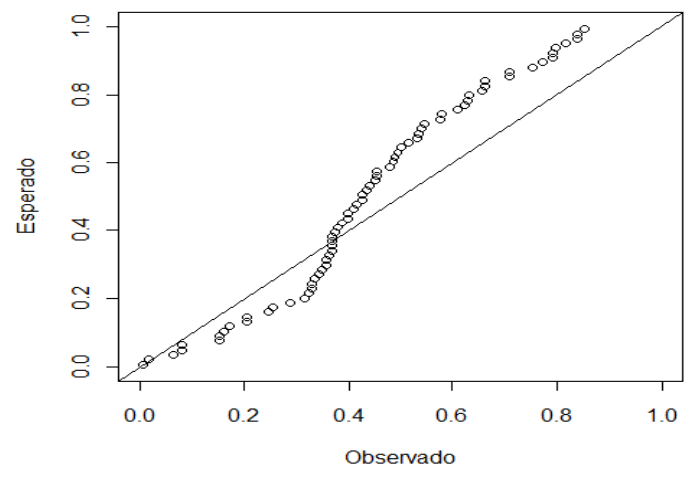

Gráfico 4 - Gráfico de probabilidade EI Fonte: Autor. 
SILVA, A. L.; RODRIGUES, J. A.; SILVA, G. O. A distribuição beta Fréchet transmutada: propriedades e aplicação

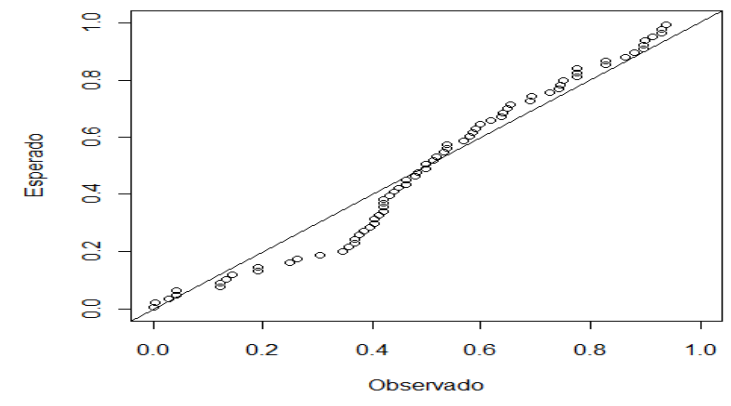

Gráfico 5 - Gráfico de probabilidade fréchet Fonte: Autor.

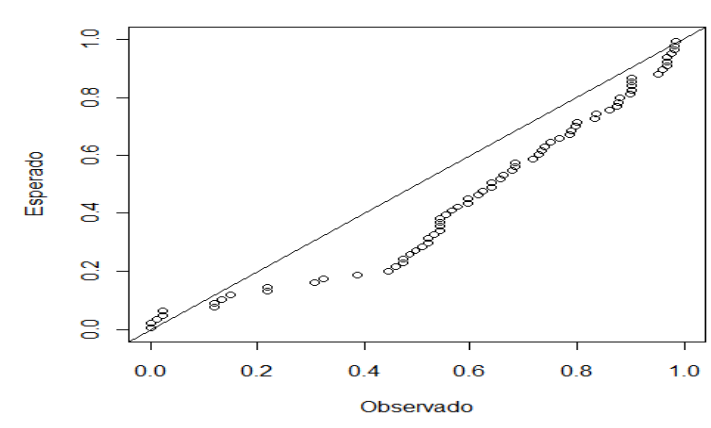

Gráfico 6 - gráfico de probabilidade ri Fonte: Autor.

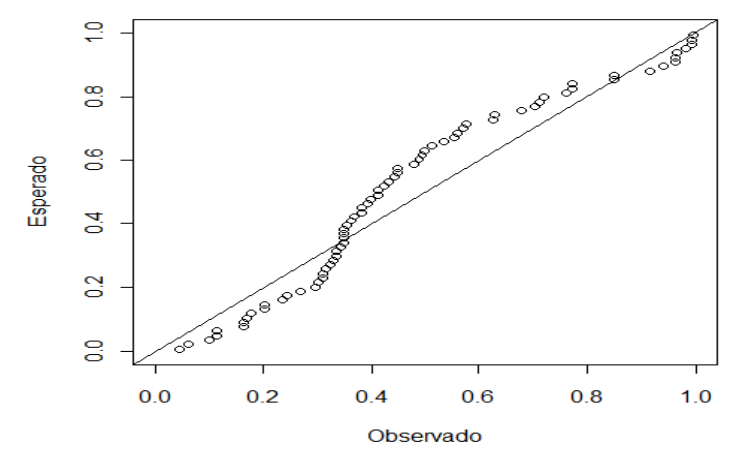

Gráfico 7 - Gráfico de probabilidade Weibull Fonte: Autor.

\section{CONCLUSÃO}

O artigo introduziu uma nova distribuição com cinco parâmetros denominada beta Fréchet transmutada. Foram deduzidas expressões para os momentos e para a função geradora de momentos. A aplicação do modelo no ajuste de um conjunto de dados correspondente aos tempos de sobrevivência, em dias, de cobaias infectadas com diferentes doses do bacilo da tuberculose revelou que o modelo proposto pode ser utilizado com sucesso na análise de dados de sobrevivência.

\section{REFERÊNCIAS}

BAHARITH, L. A et al. The beta generalized inverse Weibull distribution. British Journal of Mathematics and Computer Science, v. 4, n. 2, p. 252-270, 2014. 
SILVA, A. L.; RODRIGUES, J. A.; SILVA, G. O. A distribuição beta Fréchet transmutada: propriedades e aplicação

BJERKEDAL, T. Acquisition of resistance in guinea pigs infected with different doses of virulent tubercle bacilli. American Journal of Hygiene, v. 72, p. 130-148, 1960.

GUSMÃO, F. R. S.; ORTEGA, E. M. M.; CORDEIRO. The generalized inverse Weibull distribution. Statistical Papers, v. 52, p. 591-619, 2011.

KHAN, M. S. The beta inverse Weibull distribution. International Transactions in Mathematical Sciences and Computer, v. 3, n. 1, p. 113-119, 2010.

, KING, R. Modified inverse Weibull distribution. Journal of Statistics Applications and Probability, v. 1, n. 2, p. 115-132, 2012.

KELLER, A. Z.; KAMATH, A. R. R.; PERERA, U. D. Reliability analysis of CNC machine tools. Reliability Engineering, v. 3, n. 6, p. 449-473, 1982.

MAHMOUD, M. R.; MANDOUH, R. M. On the transmuted Fréchet distribution. Journal of Applied Sciences Research, v. 9, n. 10, p. 5553-5561, 2013.

PARARAI, M.; WARAHENA-LIYANAGE, G.; OLUYEDE, B. O. A new class of generalized inverse Weibull distribution with applications. Journal of Applied Mathematics and Bioinformatics, v. 4, n. 2, p. 17-35, 2014.

R DEVELOPMENT CORE TEAM. R: a language and environment for statistical computing. Vienna, Austria: R Foundation for Statistical Computing, 2011. Disponível em:

$<$ http://www.r-project.org>. Acesso em: 10 jun. 2014.

RISTIC, M. M.; BALAKRISHNAN, N. The gamma-exponentiated exponential distribution. Journal of Statistical Computation and Simulation, v. 82, n. 8, p. 1191-1206, 2011.

SANT'ANNA, C. C.; HIJJAR, M. A. Recente contribuição da Organização Mundial de Saúde para o controle da tuberculose na infầncia. Revista de Saúde Pública, São Paulo, v. 41, p. 117-120, 2007.

SHAHBAZ, M. Q.; SHAHBAZ, S.; BUTT, N. S. The Kumaraswamy-Inverse Weibull distribution. Pakistan Journal of Statistes and Operation Research, v. 8, n. 3, p. 479-489, 2012.

VODA, V. G. On the inverse Rayleigh distribution random variable. Report in Statistical Applied Research JUSE, v. 19, p. 13-21, 1972. 
SILVA, A. L.; RODRIGUES, J. A.; SILVA, G. O. A distribuição beta Fréchet transmutada: propriedades e aplicação

Recebido em: 11/10/2014

Aprovado em: 26/01/2015

Publicado em: 29/06/2015 